OPEN ACCESS

Edited by:

Georgina Louise Jones,

Leeds Beckett University,

United Kingdom

Reviewed by:

Jeung-Im Kim,

School of Nursing, South Korea Péter Dr. Török

University of Debrecen, Hungary Matteo Morotti,

Centre Hospitalier Universitaire

Vaudois (CHUV), Switzerland

*Correspondence: Giussy Barbara giussy.barbara@gmail.com

Specialty section

This article was submitted to

Quality of Life,

a section of the journal

Frontiers in Global Women's Health

Received: 23 June 2021 Accepted: 01 September 2021

Published: 27 September 2021

Citation:

Barbara G, Buggio L, Facchin F and Vercellini P (2021) Medical Treatment for Endometriosis: Tolerability, Quality of Life and Adherence.

Front. Glob. Womens Health 2:729601.

doi: 10.3389/fgwh.2021.729601

\section{Medical Treatment for Endometriosis: Tolerability, Quality of Life and Adherence}

\author{
Giussy Barbara ${ }^{1,2 *}$, Laura Buggio ${ }^{3}$, Federica Facchin ${ }^{4}$ and Paolo Vercellini ${ }^{2,3}$ \\ ${ }_{1}^{1}$ Obstetric and Gynaecologic Emergency Department and SVSeD (Service for Sexual and Domestic Violence), Fondazione \\ IRCCS Ca' Granda Ospedale Maggiore Policlinico, Milan, Italy, ${ }^{2}$ Department of Clinical Sciences and Community Health, \\ University of Milan, Milan, Italy, ${ }^{3}$ Gynaecology Unit, Fondazione IRCCS Ca' Granda Ospedale Maggiore Policlinico, Milan, \\ Italy, ${ }^{4}$ Department of Psychology, Catholic University of the Sacred Heart, Milan, Italy
}

Endometriosis is associated with painful symptoms, infertility, sexological difficulties, and psychological suffering. All these symptoms have a negative impact on the overall quality of life of women with the disease, with significant personal, social and economic costs. Several medical options are available to manage symptomatic endometriosis. The pharmacological treatment for endometriosis-related pain may be necessary for decades, or at least until there is a desire for pregnancy or physiologic menopause occurs. In this perspective, clinicians should consider not only the efficacy, but also side effects, tolerability, and costs, along with women's preferences toward different treatments. In this mini-review, we analyzed the pros and cons of the available drugs for the medical therapy of endometriosis, such as estrogen-progestins, progestins, GnRH agonist and $\mathrm{GnRH}$ antagonists.

Keywords: endometriosis, medical treatment, estrogen-progestins, GnRH agonists, GnRH antagonists, quality of life, tolerability

\section{INTRODUCTION}

Several medical options are available to manage symptomatic endometriosis, a chronic inflammatory estrogen-dependent disease characterized by the presence and proliferation of endometrium outside the uterine cavity (1-4). Endometriosis is associated with painful symptoms such as chronic pelvic pain, dysmenorrhea and dyspareunia, infertility, sexological difficulties, and psychological suffering (5-11). All these symptoms have a negative impact on the overall quality of life of women with endometriosis, including their social (12) and sexual relationships $(9,10,13)$, work and study productivity (14-16), with remarkable social and economic costs $(17,18)$.

The rationale underpinning medical treatment for endometriosis is that endometriotic foci respond to ovarian steroids similarly to the eutopic endometrium (19), despite the presence of endometriosis-associated peculiar endocrine pathways, such as for instance progesterone resistance $(20,21)$. Thus, from a general perspective, the different pharmacological compounds available for treating endometriosis act by interfering with the pituitary-gonadal axis, determining a hypoestrogenic state, inducing anovulation, and reducing or suppressing the amount of menstrual flow (19).

By definition, medical therapy for endometriosis is symptomatic and not curative, as the pharmacological approach is not cytoreductive and the hypo-estrogenic milieu determined by the hormonal suppression is temporary $(1,3,19)$. Consequently, the effect of the drug is expected to end 
after treatment discontinuation. However, this does not mean that medical therapy is ineffective. Like other chronic illnesses, such as hypertension or diabetes, the efficacy of pharmacological therapy on endometriosis-related symptoms should be assessed during treatment and not after discontinuation $(1,19)$.

Pelvic pain, in particular dysmenorrhea and dyspareunia, significantly affects women's experience of endometriosis and their quality of life (8). It has been demonstrated that women suffering from endometriosis-associated pelvic pain had poorer quality of life and psychological health as compared with women with asymptomatic endometriosis and the healthy controls (8).

The medical treatment of endometriosis can ameliorate painful symptoms of the disease and, consequently, reduce the negative impact on quality of life and on mental health.

In addition, it should be considered that the pharmacological treatment for endometriosis-related pain may be necessary for years, or at least until there is a desire for pregnancy or physiologic menopause occurs.

This also means that drugs for endometriosis-related pain should have a high safety profile, be well-tolerated, have few side effects, and have reasonable costs (22). Moreover, pharmacological therapies for endometriosis prevent pregnancies during their use and do not increase the likelihood of conception after their discontinuation. Therefore, women should be informed that medical therapies for endometriosis have no role in case of infertility (1). Thus, medical therapy for endometriosis should be proposed to women with endometriosis-related pain with no wish for pregnancy and without surgical indications. Absolute indications for surgery include the presence of large endometriomas, adnexal masses of uncertain appearance at diagnostic imaging procedures, ureteral stenosis causing hydronephrosis, and bowel stenosis associated with sub-occlusive symptoms (19).

According to several guidelines on endometriosismanagement released by the most authoritative gynecological societies (23-26), hormonal contraceptives, progestins, antiprogestogens, GnRH agonists, and GnRH antagonists should be used for the management of endometriosis-related pain.

A pragmatic and reasonable approach to medical treatment for endometriosis-related pain should involve a realistic balance with a long-term view between efficacy (in terms of improvement of the overall quality of life of women affected by the disease), safety, tolerability, and costs, considering women's treatment preferences and their wish for pregnancy.

\section{Estrogen-Progestins and Progestins}

Combined hormonal contraceptives have been used for many years as first-line therapy for symptomatic endometriosis. Estradiol has shown antiapoptotic and inflammatory effects on ectopic endometrial tissue, whereas progestins have antiinflammatory and pro-apoptotic properties (27).

The combined oral contraceptives currently in use contain a low level of ethinylestradiol, and have a prevalent progestin effect on ectopic endometrial tissue. In addition, they reduce or completely abolish the menstrual flow, thus limiting the amount of trans-tubaric reflux of endometrial cells. This should result in a reduction of the associated pelvic oxidative stress and inflammation induced by free peritoneal iron and heme resulting by erythrophagocytosis and lysis of erythrocytes by pelvic macrophages (28). Thus, estrogen-progestins induce atrophy of the eutopic and ectopic endometrium, limit retrograde menstruation, inhibit ovulation, and have anti-inflammatory and proapoptotic effects on endometriotic foci. Several studies (1, 19, 29-31) have shown that at least two-thirds of women affected by endometriosis-related pelvic pain will benefit from the use of estrogen-progestins combinations, in particular with regards to dysmenorrhea, with a reported significant amelioration in the overall quality of life (29-31). However, one-third of the women with endometriosis do not respond to estrogen-progestins, which may be in part due to progesterone resistance (32).

Estrogen-progestins can be delivered through different modalities $(1,33)$ - such as oral, vaginal, or transdermal according to women's preferences. The different methods of hormonal administration, which allow for a daily or weekly drug administration, can increase patients' compliance with treatment and adherence (31), which is particularly important when a pharmacological treatment is required for a long time, as is the case of a chronic disease such as endometriosis (19). In addition, estrogen-progestins represent the only treatment for endometriosis that allows the occurrence of cyclic uterine bleeding. Monthly bleeding seems important for those women who strongly believe that amenorrhea represents a nonphysiological state. Also this characteristic can increase women's compliance with treatment and adherence.

If dysmenorrhea persists with a cyclic use, women should be invited to use estrogen-progestins continuously, and to tailor the cyclic suspension of the hormonal treatment based on the occurrence of spotting/breakthrough bleeding, as well as their preferences and needs (30). An estrogen-progestin combination containing the lowest dose of estrogen and a second-generation progestin should be preferred to combine an optimal endometriosis suppression with minimization of the thrombotic risk $(1,19)$. Moreover, the most frequent adverse effects of estrogen-progestins should be explained to women, along with the other potential therapeutic alternatives (i.e., other pharmacological treatments, such as progestins, GnRH agonists and antagonists, or surgery), in order to increase women's awareness of therapeutic choices and adherence to treatment.

The European Society of Human Reproduction and Embryology (ESHRE) guidelines on endometriosis (24) pointed out that, although the evidence on the use of estrogen-progestins for endometriosis is limited, combined hormonal contraception is extensively used as a treatment for endometriosis-associated pain. This may be due to pragmatic and reasonable benefits of the estrogen-progestins therapy in women affected by endometriosis, including contraceptive action, few side effects, long term safety and good control of uterine bleeding. All these characteristics of the estrogen-progestins have a positive impact on the overall tolerability of the medical treatment and in general on patients' quality of life.

Progestins (depot medroxyprogesterone acetate, medroxyprogesterone acetate, norethisterone acetate, desogestrel and dienogest) can be used as second line treatments (1). These compounds could represent a reasonable option in women with 
endometriosis who do not respond to estrogen-progestins $(1,19)$ or in case of deep endometriotic lesions or in the presence of deep dyspareunia $(29,33,34)$. Moreover, progestins can be safely used in women with contraindications to the assumption of estrogens (35), as well as in those who do not tolerate estrogens because of their side effects (36).

Progestins can be delivered through different modalities (i.e., via oral, intramuscular, subcutaneous, or intrauterine route) to increase women's' compliance with treatment and adherence. All available progestins seem to be similarly effective in managing endometriosis-related pelvic pain and ameliorate painful symptoms and the overall quality of life in about twothirds of women affected by the disease (33). As there is no clear evidence indicating the superiority of one progestin over the others, some authors $(19,33)$ suggested using norethisterone acetate (NETA) as the first therapeutic choice because of its safety and favorable cost-effectiveness profile. In particular, oral NETA (at the dose of 2.5 and $5 \mathrm{mg}$ per day) (29, 37-43) was demonstrated to be effective in patients with deep endometriosis, such as rectovaginal or colorectal lesions $(29,38,39,43)$. In these women, the reduction in the degree of deep dyspareunia during the treatment with oral NETA was gradual but progressive over time (40).

As NETA is partially metabolized to estradiol (44), unfavorable effects on bone mineral density due to a prolonged treatment were not observed. The most frequent side effects associated with the use of NETA are weight gain, acne and seborrhea, related to the androgenic activity of this compound or to the occurrence of erratic bleeding. In case of frequent or persistent breakthrough bleeding, amenorrhea can be achieved suggesting women discontinuing treatment for some days $(30,31)$. Providing such information to women appears essential in order to improve adherence and satisfaction with therapy.

Dienogest, $2 \mathrm{mg} /$ day per os, constitutes a valid alternative in those women who experience androgenic side-effects during NETA use. In fact, due to its mild anti-androgenic properties, dienogest appears better tolerated then NETA (42), and its use may increase tolerability and treatment adherence in a large number of patients.

In general, although side effects associated with progestins are relatively frequent, about $70 \%$ of women are satisfied with this therapy. Thus, high adherence and low dropout rates have been reported with the use of progestins for the management of endometriosis-associated pain $(1,19)$.

\section{GnRH Agonists and Antagonists}

$\mathrm{GnRH}$ agonists are very effective for treating endometriosisassociated pain (24), despite their limited tolerability and safety. GnRH agonists create a deep reversible hypoestrogenic state, and evidence regarding dosage and duration of treatment is limited (24). Side effects, such as hot flushes, sleep disturbance, and mood swings, are persistent and caused by the severe hypoestrogenic state induced by these drugs. Adding an add-back therapy (i.e., low-dose progestins or tibolone) should be strongly suggested to minimize the frequency of climacteric-like symptoms and improve tolerability and adherence to therapy (24). In fact, the use of GnRH agonists as a monotherapy, especially in young women and adolescents, is limited by the unfavorable longterm safety profile, as well as by the frequency and severity of side effects.

An oral GnRH antagonist (elagolix) was recently marketed for treating women with endometriosis $(45,46)$. The mechanism of action of elagolix is a dose-dependent suppression of the ovarian estradiol production, and therefore the induction of a certain degree of hypoestrogenic state, avoiding the flare-up phase, typically associated with the use of GnRH agonists. Elagolix, at the oral daily dose of 150 or $400 \mathrm{mg}$, was found to determine a reduction in dysmenorrhea of about $46 \%$ in the lower-dose group and $76 \%$ in the higher-dose group, as compared to a menstrual pain reduction of about $23 \%$ in the placebo group (46). At 6-month follow-up, 47-66\% of women taking the higher elagolix dose of $400 \mathrm{mg}$ experienced amenorrhea. Hot flushes were reported by $42-48 \%$ of women in the $400 \mathrm{mg}$ elagolixdose group. As elagolix does not completely suppress ovulation, women should be instructed to use non-hormonal contraception. However, unplanned pregnancies were observed in women who used elagolix (46). In theory, the need for barrier contraception and the fear of unplanned pregnancies may limit women's adherence to treatment.

Other GnRH antagonists are currently being evaluated for the treatment of endometriosis, such as relugolix and linzagolix (47-50). In view of the above considerations, all these oral $\mathrm{GnRH}$ antagonists are now being studied in association with lowdose estrogen-progestin combinations, similarly to the add-back therapies used with GnRH agonists.

It would be very helpful for women with endometriosis if $\mathrm{GnRH}$ antagonists will be compared with progestins in pragmatic trials. This would allow the definition of the incremental benefit of these novel drugs compared with first-line medications not only in terms of efficacy on pain symptoms and amelioration of the women's quality of life, but also of tolerability and adherence. Moreover, the cost of any long-term medical treatment has been demonstrated to be a determinant factor for adherence (51).

\section{CONCLUSIONS}

In conclusion, when choosing medical treatments for endometriosis-related pain, clinicians should consider not only the efficacy, but also side effects, tolerability, adherence to treatment, costs and women's preferences (see also Table $\mathbf{1}$ for more details on tolerability, adherence and quality of life in the context of endometriosis). This appears. This appears particularly important if one considers the chronic nature of the disease, potentially determining a long-term impairment of women's overall quality of life, mental health, social activities, work, sexual and intimate relationships. Oral very-low-dose monophasic estrogen-progestin combinations may be considered for women with peritoneal lesions and endometriomas, whereas progestins should be favored for those with deep infiltrating lesions (1).

The heterogeneity of the studies in relation to quality of life, adherence and tolerability (in terms of research methods, types of endometriosis, and types of questionnaires used to assess quality of life outcomes) make it difficult to obtain firm 
TABLE 1 | Tolerability, quality of life, and adherence in the context of endometriosis.

Tolerability
extent to which adverse effects can be tolerated by a patient.
This could be particularly important in the management of
chronic diseases, such as endometriosis, for which treatment
may be necessary for years.
Typically, tolerability can be defined by the rate of "dropouts",
or women affected by endometriosis that forfeit participation
in a clinical study due to extreme adverse effects.
Quality of life is defined by the World Health Organization as
"individuals' perception of their position in life in the context of
the culture and value systems in which they live, and in
relation to their goals, expectations, standards and
concerns". (https://www.who.int/tools/whoqol).
Quality of life has become a major and crucial aim of current
health-care, in particular as regard to painful chronic
diseases, such as endometriosis.
Adherence to a certain pharmacological therapy refers to the
extent to which the patient's behavior fulfills with the
recommendations provided by the health care professionals.
Several factors can affect adherence to a certain treatment,
including for instance poor provider-patient communication,
fear of severe adverse effects, complex therapies that require
various medications, long-term drug regimens, economic
barriers. In the context of endometriosis, to improve woman
medication adherence, it should be important that physicians
recognize specific concerns for each woman in relation to a
certain therapy, and implement appropriate personalized
procedures to overcome them.
Adherence

conclusions. We strongly believe that researchers, in designing clinical trials in the context of endometriosis, should consider as main outcomes aspects related to women's quality of life,

\section{REFERENCES}

1. Vercellini P, Buggio L, Berlanda N, Barbara G, Somigliana E, Bosari S. Estrogen-progestins and progestins for the management of endometriosis. Fertil Steril. (2016) 106:1552-71. doi: 10.1016/j.fertnstert.2016.10.022

2. Becker CM, GattrellWT, Gude K, Singh SS. Reevaluating response and failure of medical treatment of endometriosis: a systematic review. Fertil Steril. (2017) 108:125-36. doi: 10.1016/j.fertnstert.2017.05.004

3. Ferrero S, Evangelisti G, Barra F. Current and emerging treatment options for endometriosis. Expert Opin Pharmacother. (2018) 19:110925. doi: 10.1080/14656566.2018.1494154

4. Schwartz K, Llarena NC, Rehmer JM, Richards EG, Falcone T. The role of pharmacotherapy in the treatment of endometriosis across the lifespan. Expert Opin Pharmacother. (2020) 21:893-903. doi: 10.1080/14656566.2020.1738386

5. Somigliana E, Vigano P, Barbara G, Vercellini P. Treatment of endometriosis-related pain: options and outcomes. Front Biosci. (2009) 1:455-65. doi: 10.2741/e41

6. Stratton P, Berkley KJ. Chronic pelvic pain and endometriosis: translational evidence of the relationship and implications. Hum Reprod Update. (2011) 17:327-46. doi: 10.1093/humupd/dmq050

7. Coxon L, Horne AW, Vincent K. Pathophysiology of endometriosisassociated pain: A review of pelvic and central nervous system mechanisms. Best Pract Res Clin Obstet Gynaecol. (2018) 51:53-67. doi: 10.1016/j.bpobgyn.2018.01.014

8. Facchin F, Barbara G, Saita E, Mosconi P, Roberto A, Fedele L, et al. Impact of endometriosis on quality of life and mental health: pelvic pain makes the difference. J Psychosom Obstet Gynaecol. (2015) 36:13541. doi: 10.3109/0167482X.2015.1074173 measured by validated standardized instruments to facilitate comparisons. Moreover, future studies in the pharmacological treatment of endometriosis should focus on comparison trials with other progestins or estrogen-progestins, and should be designed as superiority trials (33).

A tailored shared-decision making process should guide the clinicians in the selection of the appropriate treatment. The choice between different available therapeutic options should be pondered considering lesion type and location, the most severe reported symptom, and the need for contraception (33).

The rationale that should guide the clinicians in the management of women with endometriosis, as stated by the Practice Committee of the American Society of Reproductive Medicine (ASRM) (23), is the maximization of the use of medical therapies for long periods of time in order to achieve adequate control of pain symptoms and amelioration of quality of life, and to minimize the use of repeated surgery.

\section{AUTHOR CONTRIBUTIONS}

PV and GB conception and design of the article. GB drafting the article. GB, LB, FF, and PV literature review and acquisition of data. PV and LB revising the article for intellectual content. All authors contributed to the article and approved the submitted version.

\section{FUNDING}

This work was supported by "Ricerca Corrente 2021", Italian Ministry of Health.

9. Barbara G, Facchin F, Meschia M, Berlanda N, Frattaruolo MP, VercellinI P. When love hurts. A systematic review on the effects of surgical and pharmacological treatments for endometriosis on female sexual functioning. Acta Obstet Gynecol Scand. (2017) 96:668-87. doi: 10.1111/aogs. 13031

10. Pluchino N, Wenger JM, Petignat P, Tal R, Bolmont M, Taylor HS et al. Sexual function in endometriosis patients and their partners: effect of the disease and consequences of treatment. Hum Reprod Update. (2016) 22:76274. doi: 10.1093/humupd/dmw031

11. Gambadauro P, Carli V, Hadlaczky G. Depressive symptoms among women with endometriosis: a systematic review and meta-analysis. Am J Obstet Gynecol. (2019) 220:230-41. doi: 10.1016/j.ajog.2018.11.123

12. Culley L, Law C, Hudson N, Denny E, Mitchell H, Baumgarten M, et al. The social and psychological impact of endometriosis on women's lives: a critical narrative review. Hum Reprod Update. (2013) 19:62539. doi: 10.1093/humupd/dmt027

13. Barbara G, Facchin F, Buggio L, Somigliana E, Berlanda N, Kustermann A, et al. What Is Known and Unknown About the Association Between Endometriosis and Sexual Functioning: A Systematic Review of the Literature. Reprod Sci. (2017) 24:1566-76. doi: 10.1177/1933719117707054

14. Facchin F, Buggio L, Ottolini F, Barbara G, Saita E, Vercellini P. Preliminary insights on the relation between endometriosis, pelvic pain, and employment. Gynecol Obstet Invest. (2019) 84:190-5. doi: 10.1159/000494254

15. De Graaff AA, D'Hooghe TM, Dunselman GA, Dirksen CD, Hummelshoj L. WERF EndoCost Consortium, Simoens S. The significant effect of endometriosis on physical, mental and social wellbeing: results from an international cross-sectional survey. Hum Reprod. (2013) 28:267785. doi: 10.1093/humrep/det284 
16. Nnoaham KE, Hummelshoj L, Webster P, d'Hooghe T, de Cicco Nardone F, et al. World Endometriosis Research Foundation Global Study of Women's Health consortium. Impact of endometriosis on quality of life and work productivity: a multicenter study across ten countries. Fertil Steril. (2011) 96:366-73.e8. doi: 10.1016/j.fertnstert.2011.05.090

17. Armour M, Lawson K, Wood A, Smith CA, Abbott J. The cost of illness and economic burden of endometriosis and chronic pelvic pain in Australia: a national online survey. PLoS ONE. (2019) 1410:e0223316. doi: 10.1371/journal.pone.0223316

18. Simoens S, Dunselman G, Dirksen C, Hummelshoj L, Bokor A, Brandes I, et al. The burden of endometriosis: costs and quality of life of women with endometriosis and treated in referral centres. Hum Reprod. (2012) 27:12929. doi: 10.1093/humrep/des073

19. Vercellini P, Buggio L, Frattaruolo MP, Borghi A, Dridi D, Somigliana E. Medical treatment of endometriosis-related pain. Best Pract Res Clin Obstet Gynaecol. (2018) 51:68-91. doi: 10.1016/j.bpobgyn.2018.01.015

20. Patel BG, Rudnicki M, Yu J, Shu Y, Taylor RN. Progesterone resistance in endometriosis: origins, consequences and interventions. Acta Obstet Gynecol Scand. (2017) 96:623-32. doi: 10.1111/aogs.13156

21. Reis FM, Coutinho LM, Vannuccini S, Batteux F, Chapron C, Petraglia F. Progesterone receptor ligands for the treatment of endometriosis: the mechanisms behind therapeutic success and failure. Hum Reprod Update. (2020) 26:565-85. doi: 10.1093/humupd/dma a009

22. Dworkin RH, Turk DC, McDermott MP, Peirce-Sandner S, Burke LB, Cowan $\mathrm{P}$, et al. Interpreting the clinical importance of group differences in chronic pain clinical trials: IMMPACT recommendations. Pain. (2009) 146:238e44. doi: 10.1016/j.pain.2009.08.019

23. Practice Committee of the American Society for Reproductive Medicine. Treatment of pelvic pain associated with endometriosis: a committee opinion. Fertil Steril. (2014) 101:927-35. doi: 10.1016/j.fertnstert.2014.02.012

24. Dunselman GAJ, Vermeulen N, Becker C, Calhaz-Jorge C, D’Hooghe T, De Bie B, et al. ESHRE guideline: management of women with endometriosis. Hum Reprod. (2014) 29:400-12. doi: 10.1093/humrep/det457

25. American College of Obstetricians and Gynecologists. Management of endometriosis. ACOG practice bulletin no. 114. Obstet Gynecol. (2010) 116:223-36. doi: 10.1097/AOG.0b013e3181e8b073

26. Leyland N, Casper R, Laberge P, Singh SS. Society of obstetricians and gynaecologists of Canada. Endometriosis: Diagnosis and management. J Obstet Gynecol Can. (2010) 32:S1-S32. doi: 10.1016/S1701-2163(16) 34589-3

27. Reis FM, Petraglia F, Taylor RN. Endometriosis: hormone regulation and clinical consequences of chemotaxis and apoptosis. Hum Reprod Update. (2013) 19:406-18. doi: 10.1093/humupd/dmt010

28. Donnez J, Binda MM, Donnez O, Dolmans MM. Oxidative stress in the pelvic cavity and its role in the pathogenesis of endometriosis. Fertil Steril. (2016) 106:1011-7. doi: 10.1016/j.fertnstert.2016.07.1075

29. Vercellini P, Pietropaolo G, De Giorgi O, Pasin R, Chiodini A, Crosignani PG. Treatment of symptomatic rectovaginal endometriosis with an estrogen-progestogen combination versus low-dose norethindrone acetate. Fertil Steril. (2005) 84:1375-87. doi: 10.1016/j.fertnstert.2005. 03.083

30. Vercellini P, Frontino G, De Giorgi O, Pietropaolo G, Pasin R, Crosignani PG. Continuous use of an oral contraceptive for endometriosisassociated recurrent dysmenorrhea that does not respond to a cyclic pill regimen. Fertil Steril. (2003) 80:560-3. doi: 10.1016/S0015-0282(03) 00794-5

31. Vercellini P, Barbara G, Somigliana E, Bianchi S, Abbiati A, Fedele L. Comparison of contraceptive ring and patch for the treatment of symptomatic endometriosis. Fertil Steril. (2010) 93:2150-61. doi: 10.1016/j.fertnstert.2009.01.071

32. Donnez J, Dolmans MM. Endometriosis and medical therapy: from progestogens to progesterone resistance to $\mathrm{GnRH}$ antagonists: a review. J Clin Med. (2021) 10:1085. doi: 10.3390/jcm10051085

33. Buggio L, Somigliana E, Barbara G, Frattaruolo MP, Vercellini P. Oral and depot progestin therapy for endometriosis: towards a personalized medicine. Expert Opin Pharmacother. (2017) 18:1569-81. doi: 10.1080/14656566.2017.1381086
34. Casper RF. Progestin-only pills may be a better first-line treatment for endometriosis than combined estrogen-progestin contraceptive pills. Fertil Steril. (2017) 107:533-6. doi: 10.1016/j.fertnstert.2017. 01.003

35. Berlanda N, Somigliana E, Viganò P, Vercellini P. Safety of medical treatments for endometriosis. Expert Opin Drug Saf. (2016) 15:2130. doi: $10.1517 / 14740338.2016 .1121991$

36. Morotti M, Remorgida V, Venturini PL, Ferrero S. Progestogen-only contraceptive pill compared with combined oral contraceptive in the treatment of pain symptoms caused by endometriosis in patients with migraine without aura. Eur J Obstet Gynecol Reprod Biol. (2014) 179:638. doi: 10.1016/j.ejogrb.2014.05.016

37. Muneyyirci-Delale O, Karacan M. Effect of norethindrone acetate in the treatment of symptomatic endometriosis. Int J Fertil Women's Med. (1998) 43:24e7.

38. Ferrero S, Camerini G, Ragni N, Venturini PL, Biscaldi E, Remorgida V. Norethisterone acetate in the treatment of colorectal endometriosis: a pilot study. Hum Reprod. (2010) 25:94e100. doi: 10.1093/humrep/ dep361

39. Ferrero S, Camerini G, Ragni N, Venturini PL, Biscaldi E, Seracchioli R, et al. Letrozole and norethisterone acetate in colorectal endometriosis. Eur J Obstet Gynecol Reprod Biol. (2010) 150:199e202. doi: 10.1016/j.ejogrb.2010. 02.023

40. Vercellini P, Somigliana E, Consonni D, Frattaruolo MP, De Giorgi O, Fedele L. Surgical versus medical treatment for endometriosis-associated severe deep dyspareunia: I. Effect on pain during intercourse and patient satisfaction. Hum Reprod. (2012) 27:3450e9. doi: 10.1093/humrep/ des313

41. Vercellini P, Frattaruolo MP, Somigliana E, Jones GL, Consonni D, Alberico $\mathrm{D}$, et al. Surgical versus low-dose progestin treatment for endometriosisassociated severe deep dyspareunia II: effect on sexual functioning, psychological status and health-related quality of life. Hum Reprod. (2013) 28:1221e30. doi: 10.1093/humrep/det041

42. Vercellini P, Bracco B, Mosconi P, Roberto A, Alberico D, Dhouha $\mathrm{D}$, et al. Norethindrone acetate or dienogest for the treatment of symptomatic endometriosis: a before and after study. Fertil

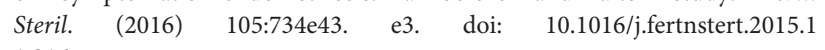
1.016

43. Morotti M, Venturini PL, Biscaldi E, Racca A, Calanni L, Vellone VG, et al. Efficacy and acceptability of long-term norethindrone acetate for the treatment of rectovaginal endometriosis. Eur J Obstet Gynecol Reprod Biol. (2017) 213:4e10. doi: 10.1016/j.ejogrb.2017.0 3.033

44. Chu MC, Zhang X, Gentzschein E, Stanczyk FZ, Lobo RA. Formation of ethinyl estradiol in women during treatment with norethindrone acetate. J Clin Endocrinol Metab. (2007) 92:2205e7. doi: 10.1210/jc.20070044

45. Carr B, Dmowski WP, O’Brien C, Jiang P, Burke J, Jimenez R, et al. Elagolix, an oral $\mathrm{GnRH}$ antagonist, versus subcutaneous depot medroxyprogesterone acetate for the treatment of endometriosis: effects on bone mineral density. Reprod Sci. (2014) 21:1341e51. doi: 10.1177/193371911454 9848

46. Taylor HS, Giudice LC, Lessey BA, Abrao MS, Kotarski J, Archer DF, et al. Treatment of endometriosis-associated pain with elagolix, an oral GnRH antagonist. N Engl J Med. (2017) 377:28e40. doi: 10.1056/NEJMoa1 700089

47. Osuga Y, Seki Y, Tanimoto M, Kusumoto T, Kudou K, Terakawa N. Relugolix, an oral gonadotropin-releasing hormone receptor antagonist, reduces endometriosis-associated pain in a dose-response manner: a randomized, double-blind, placebo-controlled study. Fertil Steril. (2021) 115:397-405. doi: 10.1016/j.fertnstert.2020.07.055

48. Osuga Y, Seki Y, Tanimoto M, Kusumoto T, Kudou K, Terakawa N. Relugolix, an oral gonadotropin-releasing hormone $\mathrm{GnRH}$ receptor antagonist, in women with endometriosis-associated pain: phase 2 safety and efficacy 24-week results. BMC Womens Health. (2021) 21:250. doi: 10.1186/s12905-021-01393-3

49. Donnez J, Taylor HS, Taylor RN, Akin MD, Tatarchuk TF, Wilk K, et al. Treatment of endometriosis-associated pain with linzagolix, an oral 
gonadotropin-releasing hormone-antagonist: a randomized clinical trial. Fertil Steril. (2020) 114:44-55. doi: 10.1016/j.fertnstert.2020.02.114

50. Borini A, Coticchio G. Gonadotropin-releasing hormone antagonist linzagolix: possible treatment for assisted reproduction patients presenting with adenomyosis and endometriosis? Fertil Steril. (2020) 114:517-8. doi: 10.1016/j.fertnstert.2020.06.003

51. Vercellini P, Facchin F, Buggio L, Barbara G, Berlanda N, Frattaruolo MP, et al. Management of endometriosis: toward value-based, cost-effective, affordable care. J Obstet Gynaecol Can. (2018) 40:726-749.e10. doi: 10.1016/j.jogc.2017.07.011

Conflict of Interest: The authors declare that the research was conducted in the absence of any commercial or financial relationships that could be construed as a potential conflict of interest.
Publisher's Note: All claims expressed in this article are solely those of the authors and do not necessarily represent those of their affiliated organizations, or those of the publisher, the editors and the reviewers. Any product that may be evaluated in this article, or claim that may be made by its manufacturer, is not guaranteed or endorsed by the publisher.

Copyright $\odot 2021$ Barbara, Buggio, Facchin and Vercellini. This is an open-access article distributed under the terms of the Creative Commons Attribution License (CC $B Y)$. The use, distribution or reproduction in other forums is permitted, provided the original author(s) and the copyright owner(s) are credited and that the original publication in this journal is cited, in accordance with accepted academic practice. No use, distribution or reproduction is permitted which does not comply with these terms. 